\title{
Promoção da saúde da mulher: representação social de enfermeiras
}

\section{Promotion of women's health: social representation of nurses}

DOI: $10.46919 / \operatorname{archv} 1 \mathrm{n} 5-010$

Recebimento dos originais: 10/07/2020

Aceitação para publicação: 30/08/2020

Victória Agna Alves Nascimento da Silva

Enfermeira pelo Centro Universitário de Volta Redonda - Unifoa. Pós graduanda em Gestão estratégica da saúde da família pela universidade Faveni.

Instituição: Centro Universitário de Volta Redonda - UniFOA

Endereço: Av. Paulo Erlei Alves Abrantes, 1325, Volta Redonda (RJ), Brasil.

E-mail: enfvictoriaagna@outlook.com

\section{Renata Martins da Silva Pereira}

Doutora em Ciências pela Universidade Federal do Estado do Rio de Janeiro (UNIRIO)

Instituíção: Universidade do Estado do Rio de Janeiro (UERJ)/ Centro Universitário de Volta Redonda (UniFOA).

Endereço: Av. Paulo Erlei Alves Abrantes, 1325. Volta Redonda (RJ), Brasil.

E-mail: renataenfprofessora@gmail.com

\section{Clarissa Ferreira Pontual de Oliveira}

Mestre em Ciências da Saúde e Meio Ambiente pelo Centro Universitário de Volta Redonda - UniFOA.

Instituição: Centro Universitário de Volta Redonda - UniFOA

Endereço: Av. Paulo Erlei Alves Abrantes, 1325, Volta Redonda (RJ), Brasil.

E-mail: pontualclarissa@gmail.com

\section{Dyene kelly Leopoldina Rodrigues da Silva}

Enfermeira pelo Centro Universitário de Volta Redonda - UniFOA.

Pós-graduanda no Centro Universitário de Volta Redonda - UniFOA

Instituição: Centro Universitário de Volta Redonda - UniFOA

Endereço: Av. Paulo Erlei Alves Abrantes, 1325. Volta Redonda (RJ), Brasil.

E-mail: dyenekelly.09@gmail.com

\section{RESUMO}

A enfermeira no cotidiano de seu trabalho tem a responsabilidade de cuidado do indivíduo, da família e de toda comunidade. Ela é responsável, junto à equipe multiprofissional, por ampliar o acesso da população de igualitária aos serviços de saúde. Para isto conta não somente com conhecimento clínico, mas também com a responsabilidade social para impulsionar mudanças de comportamento frente a manutenção da saúde de mulheres assistidas. Propiciando uma atenção em que haja a predominância da promoção da autonomia da usuária sobre sua saúde e prevenção de agravos. O objetivo da pesquisa foi identificar a representação social atribuídas pelas enfermeiras ao seu cuidado destinado a mulheres na atenção básica. Trata-se de uma pesquisa de campo com abordagem qualitativa do tipo descritiva e que utilizou a Teoria das Representações Sociais para a análise dos dados. Foi desenvolvida no município de Angra dos Reis (RJ) em Unidades de Estratégia de Saúde da Família. Foram entrevistadas 10 enfermeiras. Os resultados demonstraram que $90 \%$ das participantes se sentem realizadas com sua profissão. $70 \%$ disseram ter papel importante como promotora do cuidado e se sentem como referência para a promoção 
do cuidado, apesar de encontrar obstáculos culturais e de questões de gênero para praticar o cuidado às mulheres. Conclui-se que a formação de vínculo e o olhar minucioso para as necessidades de cada mulher individualmente pode promover um cuidado qualificado e gerar satisfação tanto às clientes quanto às enfermeiras na prática de sua profissão.

Palavras-chave: Saúde da mulher, Atenção primária, Enfermagem.

\begin{abstract}
The nurse in her daily work has the responsibility to care for the individual, the family and the whole community. She is responsible, together with the multiprofessional team, for expanding the access of the egalitarian population to health services. For this, it relies not only on clinical knowledge, but also on social responsibility to drive behavioral changes in the face of maintaining the health of assisted women. Providing care in which there is a predominance of promoting the user's autonomy over their health and preventing injuries. The objective of the research was to identify the social representation attributed by nurses to their care for women in primary care. It is a field research with a qualitative approach of the descriptive type and which used the Theory of Social Representations for data analysis. It was developed in the city of Angra dos Reis (RJ) in Family Health Strategy Units. 10 nurses were interviewed. The results showed that $90 \%$ of the participants feel fulfilled with their profession. $70 \%$ said they play an important role as a promoter of care and feel they are a reference for the promotion of care, despite encountering cultural and gender obstacles to practice care for women. It is concluded that the formation of bonds and a detailed look at the needs of each individual woman can promote qualified care and generate satisfaction for both clients and nurses in the practice of their profession.
\end{abstract}

Keywords: Women's health, Primary attention, Nursing.

\title{
1 INTRODUÇÃO
}

A enfermeira no cotidiano de seu trabalho tem a responsabilidade de cuidado do indivíduo, da família e de toda comunidade. Ela é responsável, junto à equipe multiprofissional, por ampliar o acesso da população de igualitária aos serviços de saúde. Para isto conta não somente com conhecimento clínico, mas também com a responsabilidade social para impulsionar mudanças de comportamento frente a manutenção da saúde de mulheres assistidas. Propiciando uma atenção em que haja a predominância da promoção da autonomia da usuária sobre sua saúde e prevenção de agravos.

A proposta do trabalho desenvolvido pela estratégia de saúde da família "elege como ponto central o estabelecimento de vínculos e a criação de laços de compromisso e de corresponsabilidade entre os profissionais de saúde e a população" (1). A enfermeira em seu cotidiano de orientação, principalmente no cuidado a mulher, tem uma responsabilidade grande, porque conforme as orientações chegam a mulher, a enfermeira vai contribuir indiretamente para a saúde de toda família.

As mulheres constituem a maioria da população brasileira e são as principais usuárias do Sistema Único de Saúde (SUS). Conformam, portanto, um segmento social fundamental para as políticas de saúde, especialmente porque as históricas desigualdades de poder entre mulheres e homens implicam em forte impacto nas condições de saúde das mulheres. O trabalho da enfermeira com a mulher, quando bem 
realizado, permite a esta o poder de decisão e faz com que a mulher se veja como cidadã e autora de sua própria história de saúde, contribuindo assim para que cada vez mais mulheres participem de seu autocuidado com conhecimento e autonomia sobre seu corpo e seus direitos à saúde emocional e clínica, reprodutiva, sexual, psiquiátrica, oncológica, preventiva e esportiva, de forma imponderada e no tempo oportuno como preconizado pelo Ministério da Saúde ${ }^{(2)}$.

Cabe a enfermeira no âmbito de saúde da família e atendimento a mulher fazer abordagem dos problemas/queixas e prevenções dos cânceres que mais acometem a população feminina, pré-natal, puerpério e aleitamento materno, planejamento reprodutivo, climatério e atenção às mulheres em situação de violência doméstica e sexual ${ }^{(3)}$.

A teoria das representações sociais (TRS) proposta por Serge Moscovici é uma abordagem que tem sido muito utilizada nas pesquisas de enfermagem e através dela busca-se conceitos e afirmações que surgem no cotidiano através da comunicação e vivência interindividuais, relacionando-as e contribuindo para a prática e conduta de enfermeiras ${ }^{(4) .}$ Esse tipo de pesquisa permite não somente o reconhecimento das práticas do grupo, mas faz também com que aconteça intervenções mais eficientes respeitando as características especificas de cada paciente ${ }^{(5)}$.

As representações sociais são uma modalidade de conhecimento particular entre indivíduos em que esta teoria trabalha com o cognitivo do indivíduo e sua interação no meio social atuando na transformação do mesmo, reconhece como um grupo reapresenta e constrói uma realidade ${ }^{(6)}$.

As representações sociais permitem a compreensão da realidade por meio do senso comum, tido no acadêmico como saber ingênuo, permitindo a integração do conhecimento advindo de atores sociais a um quadro que se faz compreensível para eles mesmos, sendo justaposto ao funcionamento cognitivo e aos valores aos quais se ajuntam ${ }^{(6)}$.

Neste contexto a pesquisa em enfermagem relacionando a TRS e saúde da mulher busca compreender como a enfermeira se vê frente as responsabilidades no cuidado com a mulher, o que a enfermeira pensa sobre esse cuidado e como isso pode modificar a saúde dessas mulheres e de seus familiares.

Diante do exposto, o objetivo da pesquisa foi identificar a representação social atribuída pelas enfermeiras ao seu cuidado destinado a mulheres na atenção básica.

\section{METODOLOGIA}

Trata-se de uma pesquisa de campo com abordagem qualitativa do tipo descritiva. O estudo foi desenvolvido no município de Angra dos Reis (RJ) em Unidades de Estratégia de Saúde da Família. Onde as participantes foram estimuladas a dar sua opinião sobre o objetivo desse trabalho, as perguntas foram 
feitas de modo que as respostas fossem dadas de modo aberto e deixado as entrevistadas à vontade para responder com base exclusivamente naquilo que pensa.

Participaram da pesquisa 10 enfermeiras que fazem atendimento a mulheres nas unidades de Estratégia de Saúde da família (ESF) no municio de Angra dos Reis, e que atenderam ao critério de inclusão de ao menos ter 1 ano de experiência em serviço na atenção básica, além de uma carga horária de trabalho de acima de 30 horas semanais, eram ainda responsáveis pelo trabalho assistencial e/ou gerencial, de forma que este perfil atende os pressupostos de cuidado a mulher que poderão fortalecer os resultados do estudo. Foram excluídos as enfermeiras que se encontravam afastadas das atividades por férias e licenças.

Quanto as unidades de Estratégia de Saúde da família fizeram parte do cenário de pesquisa 10 pontos de atendimento da ESF. Cada unidade possui uma equipe multidisciplinar composta por 1 enfermeiro, 1 auxiliar de enfermagem, 1 médico, 1 dentista, 1 auxiliar de saúde bucal e até 6 agentes comunitários de saúde.

A coleta de dados ocorreu no mês de agosto de 2018 e foi feita através da técnica de entrevista semiestruturada, contemplando questionamentos relacionados à vivencias das enfermeiras acerca da rotina e das atividades desenvolvidas nos serviços que estavam inseridas. As entrevistas foram agendadas de acordo com a disponibilidade das participantes em relação à data, horário e local. As entrevistas foram gravadas por meio de um gravador portátil de voz e, por sua vez, transcritas na íntegra através do aplicativo dictate.

Nas transcrições, cada participante foi identificada pela letra "E" seguido de codificação alfanumérica, de acordo com a sequência da realização das entrevistas (E1, E2, E3...).

A análise de dados seguiu a abordagem qualitativa baseada na Teoria das Representações Sociais de Serge Moscovici onde entende-se que as Representações Sociais unem o sujeito ao objeto, o pensamento à ação, a razão à emoção, o individual ao coletivo; logo, estudar o cuidado às mulheres pela via das representações sociais abre inúmeras possibilidades de compreensão não somente das ações das enfermeiras no âmbito da saúde, mas dos sentidos que elas atribuem a essas ações em face dos contextos em que elas são produzidas, justificando suas opções frente às realidades que se lhes apresentam. ${ }^{\text {(5) }}$

E o tratamento dos dados transcritos foi feito pela análise de conteúdo proposta por Bardin, onde utiliza-se um conjunto de técnicas de análise das comunicações visando a obter, por procedimentos sistemáticos e objetivos de descrição do conteúdo das mensagens, através de pré-análise, exploração do conteúdo apresentado e interpretação para formação de categorias ${ }^{(7)}$. 
O estudo foi aprovado sob Parecer $n^{\circ}$ 2.761.539seguindo os preceitos éticos descritos na resolução $n^{\circ}$ 466/ 2012 do Conselho Nacional de Saúde- CNS, a qual regulamenta os aspectos legais para Pesquisas com Seres Humanos. As participantes assinaram o Termo de Consentimento Livre e Esclarecido.

\section{RESULTADOS}

Em relação a caracterização das participantes, quanto a idade variou entre 31 e 41 anos, com média de 35 anos de idade. O tempo de formação ficou entre 10 e 15 anos, com média de 11,5 anos de formadas.

Quanto a especialização citada pelas participantes, observou-se que houve variação nas especialidades. Das 10 participantes, 8 tem curso de pós-graduação em saúde da família, assim também como 4 delas apresentam formação em Gestão em Enfermagem, e ainda foram citadas Nefrologia, Enfermagem do trabalho, Segurança do paciente, Formação pedagógica do SUS e Estomaterapia. Vale destacar que todas as participantes tinham pós-graduação lato sensu.

Quanto a atuação por horas semanais e em anos trabalhados, observou-se que $80 \%$ das enfermeiras cumpre uma jornada de trabalho de 40 horas semanais e $20 \%$ pelo menos 30 horas por semana. As participantes têm experiência no serviço, com média de 8 anos e 5 meses de atuação em atenção básica, variando entre 4 e 13 anos de serviço.

90\% das participantes responderam que se sentem realizadas com sua profissão, como pode ser destacado nas falas abaixo:

\footnotetext{
Sinto que meu trabalho faz diferença na vida das pessoas e que levo qualidade de vida para elas, isso faz com que eu me sinta importante. (EA 4)

É uma área que eu gosto, é uma grande responsabilidade, mas vejo que a população tem necessidade do meu trabalho, mas acredito que a classe precisa melhorar. "Me sinto bem sendo enfermeira". (EA 7)

Percebo que a enfermagem da atenção básica consegue fazer diferença na saúde dos indivíduos com ações simples e ao mesmo tempo complexas. "A vezes penso que poderia contribuir mais, mas algumas coisas fogem da nossa governabilidade". (EA 9)
}

Quando questionadas sobre seu papel de promotora do cuidado a saúde de mulheres atendidas por elas, das 10 entrevistadas 70\% disseram ter papel importante como promotora do cuidado e se sentem como referência para a promoção do cuidado, apesar de encontrar obstáculos culturais/de cunho profissional.

\footnotetext{
"Na esf (estratégia de saúde da família), a gente atende uma grande parte do programa de saúde da mulher e eu vejo que nós temos um papel muito importante, apesar de ainda esbarrar em barreiras culturais como: A é a enfermeira que vai atender?" (EA 1)

"Muito importante, mas muito difícil de acontecer por questões da vida da mulher, de como ela está inserida na sociedade, se trabalha. Fundamental porque vai interferir na vida da mulher e
} 
porque ela precisa entender as questões da sua saúde como métodos contraceptivos, a questão da reprodução, os exames." (EA2)

"Papel muito importante, principalmente onde tem médicos homens, que a maioria das pacientes querem ser atendidas por mulheres. O contato mulher com mulher é muito importante e o trabalho da enfermeira essencial."(EA10) .

"É um trabalho de grande responsabilidade..." Eu me vejo como promotora do cuidado a saúde da mulher pois nós somos a referência que elas têm na unidade, e por ser referência mesmo que a mulher tenha outras demandas que não sejam da área de enfermagem acabamos nos envolvendo e sendo a porta de entrada para todos os cuidados das pacientes.(EA6)"

$20 \%$ expressaram que acreditam poder interferir no processo de empoderamento da mulher, no que se refere ao cuidar de si própria, como pode ser destacado nas falas abaixo:

"Sinto que sou responsável pelo empoderamento da mulher no quesito autocuidado e de fazer com que a mulher perceba que ela é a responsável por sua saúde. “Com o meu trabalho quero que ela entenda que é a única responsável pela sua saúde, claro que depende de profissionais e do serviço, mas ela é responsável por se cuidar". "Quando a mulher tem conhecimento da sua saúde ela influencia também toda a família, a mulher é a porta de entrada na família na maioria das vezes".(EA9).

"Acabamos interferindo para que a mulher se cuide em diversas áreas de sua vida não apenas na saúde, mas também quanto ao seu papel na sociedade, trabalhamos também com a questão do empoderamento da mulher, e isso ajuda muito a vida dessas mulheres.’(EA5)

$10 \%$ das enfermeiras entrevistadas disseram ter o sentimento de realização de um trabalho gratificante, por poder participar da vida das mulheres e de sua família atuando na promoção de sua saúde.

"É um papel gratificante e interfere diretamente no processo de saúde e doença da mulher, porque a mulher é mais atuante dentro da unidade de saúde, é quem mais participa e é ela que traz a família para o atendimento, então quando criamos vínculo com a mulher acabamos nos aproximando do ambiente familiar como um todo.(EA5)"

Já quando questionadas sobre como podem auxiliar as mulheres a serem mais conscientes sobre sua saúde e questões de prevenção, constatou-se que das 10 entrevistadas, $100 \%$ citaram a formação do vínculo com essas mulheres como indutor de instrução, educação em saúde e transformação da realidade das mulheres atendidas.

"Acredito no trabalho em consultório de forma sequenciada. Se a mulher passa confiar em mim, ela vai voltar e vai criar um vínculo que pode melhorar e modificar a vida dela ou de repente não, mas ela vai pensar. É um trabalho de formiguinha, mas que você consegue ver resultado. '(EA2)

"Trabalho de formiguinha, difícil e de acolher. Quanto melhor eu acolho essa mulher mais sensível ela vai estar a qualquer informação ou discussão a nível de consultório ou até mesmo a nível de grupo. O acolhimento é a forma mais real para auxiliar essa mulher na melhor adesão ao cuidado." (EA 3)

No meu trabalho especificamente existe uma questão de vinculo e confiança com as pacientes, isso me proporciona uma certa autonomia no trabalho, e aqui tenho condições de ter retorno das pacientes, devido a credibilidade que temos e mesmo com todas as dificuldades que encontramos no SUS elas confiam e sabem que os profissionais estão fazendo a diferença. (EA6) 
"Falando do cotidiano, dando exemplos, fazendo pesquisas, educação em saúde, conversando e com trabalhos práticos. ...Saúde da mulher é uma das áreas que mais tem me dado alegria. Sinto que tenho a confiança dessas mulheres e que contribuo para a promoção do cuidado. Sinto que tenho um papel efetivo, diferente de outros grupos. É a única parte prazerosa do meu trabalho hoje."(EA4)

"O atendimento da mulher é o que mais dar resultado, é o que mais dar retorno na saúde da família. Eu percebo que com a mulher a gente se identifica mais e consegue perceber elas de uma forma mais fácil... na comunicação não verbal. A gente consegue atingir um plano de cuidado melhor e por entender o lado de ser mulher."(EA3)

Quanto a responsabilidade frente a saúde do público feminino, as 10 enfermeiras referem-se as competências próprias do exercício profissional de Enfermagem profissão como atenção à saúde e a comunicação empática.

\footnotetext{
' Me vejo com uma grande e importante responsabilidade, porque o público feminino precisa saber que aqui na unidade tem o cuidado que elas precisam, e mesmo com muitas dificuldades tentamos garantir um bom atendimento às pacientes, pois temos o compromisso de promover a saúde. "(EA6)

" Promotora de mudança e não posso ser indiferente frente a realidade social e política. Sinto que posso fazer a diferença na vida dessas mulheres." (EA4)

"Sou a promotora de saúde daquele território e o que tento fazer é trazer mais informações para essa mulher para que ela tenha mais consciência das suas ações. Tenho que fazer com que aquela mulher tenha autonomia sobre o organismo dela." (EA8)

"Deixar a mulher falar e ai sim tentar uma intervenção. Quando a gente ouve a gente assume uma responsabilidade grande. Temos que ter empatia na saúde da mulher, cuidado, as coisas geram em torno da fala.',(EA2)
}

Quando questionadas sobre quais as dificuldades encontradas para que o público feminino siga as recomendações oferecidas durante as consultas, $90 \%$ das enfermeiras disseram que a maior dificuldade ainda é a questão de gênero que permeia as relações humanas em sociedade, como a falta de apoio dos parceiros que são resistentes aos métodos, que não aderem ao tratamento dificultando deste modo a eficácia do mesmo e levando a recidiva de certas doenças.

\begin{abstract}
"Educação, parceiros quase não vem a unidade e atrapalha o tratamento porque algumas vezes vai contra ao que estamos falando. ',(EA2)

"tem uma dificuldade muito grande da aceitação do parceiro e do cultural também. Na minha comunidade tenho um público feminino mais idoso e com pouco conhecimento, é difícil colocar algumas informações na cabeça delas e tirar os mitos ou ir contra o que marido acredita. " (EA8)
\end{abstract}

Ainda foram citadas a falta de insumos, medicamentos, recursos humanos, estruturação do ambiente e a questão cultural da população.

"Falta de insumos, falta de recursos humanos e infelizmente o baixo nível cultural da população." (EA 1)

"A falta de material prejudica bastante nosso atendimento e isso me traz uma certa indignação, pois sabemos que isso é devido à má administração dos recursos públicos e que existem outras prioridades para os recursos, mas infelizmente não podemos fazer nada quanto a isso. '’(EA 6) 


\section{DISCUSSÃO}

O sentimento de realização profissional no trabalho em enfermagem interfere diretamente na qualidade do cuidado prestado aos pacientes. Satisfação com o trabalho é um conjunto de sentimentos favoráveis que os indivíduos apresentam em relação ao mesmo, e quanto maiores forem os fatores de satisfação, maior poderá ser o empenho do profissional em prestar uma assistência qualificada, refletindo um serviço de melhor qualidade ${ }^{(8)}$. Desta forma fica claro, assim como na presente pesquisa, que a satisfação ao cuidar das mulheres atendidas pelas enfermeiras traz para as participantes sentimentos positivos com o trabalho e que interferem na tomada de posição frente a qualidade dos serviço prestado por estas.

Essa satisfação é a somatória de diferentes elementos que compõem o mundo pessoal e profissional, bem como o resultado da avaliação que o trabalhador tem acerca de seu trabalho (8). Destacando-se um conjunto de elementos que contribuem para que haja satisfação, como: o reconhecimento, a felicidade, o respeito, o ambiente de trabalho e as condições de trabalho.

O cuidado é o princípio do trabalho da enfermagem e na atenção básica, principalmente, a enfermeira tem um papel importante de cuidar e promover o cuidado dos pacientes dentro das suas limitações. Nessa pesquisa as enfermeiras dizem que se reconhecem como promotoras do cuidado a saúde das mulheres que atendem, relatam ainda que nessa promoção de cuidado esbarram muitas vezes em dificuldades culturais e sociais. No que se refere à saúde da mulher, é fundamental que se conheça sua cultura, pelo simples fato de que suas crenças e determinados saberes populares estarem intimamente ligados com o conhecimento de antepassados que vão interferir no autocuidado e na prática em saúde, tanto da mulher como da sua família ${ }^{(9)}$.

Esses aspectos culturais da relação enfermeira-paciente, proporcionam troca de conhecimento de forma sensível e ajuda o outro a crescer. A enfermeira, ao cuidar, está se desenvolvendo profissional e pessoalmente. No âmbito da saúde da mulher, essa relação está impregnada de valores, crenças e atitudes que nascem e se transformam no convívio social e da comunidade ${ }^{(9)}$.

Como estratégia para uma promoção efetiva do cuidado todas as participantes da pesquisa relataram criar vínculos com as mulheres atendidas pelas mesmas, sendo instrumento indutor de instrução, educação em saúde e transformação da realidade das mulheres atendidas. Dar voz às mulheres nos serviços de saúde permite o conhecimento de suas histórias e a elaboração de ações que atendam às suas reais necessidades, pois, assim como a enfermagem exerce influência na saúde da mulher, esta também interfere na enfermagem com suas concepções de mundo ${ }^{(9)}$. É com base no conhecimento das realidades individuais e sociais que a enfermagem busca embasamentos para o planejamento das ações do 
seu cuidar. E se entendemos que cuidar é um ato social, a busca do equilíbrio dessa relação traz, também, um equilíbrio social, tendo assim, um potencial de transformação das realidades assistenciais vigentes.

Algumas enfermeiras citaram que durante atendimento à mulher elas buscam realizar o empoderamento feminino, desenvolvendo conhecimento e fortalecendo a participação dessa mulher no cuidado da sua saúde.

De acordo com a tradução brasileira, o termo empoderar compreende em dar ou adquirir poder ou mais poder. As mulheres tornam-se empoderadas por meio da tomada de decisões coletivas e de mudanças individuais. O empoderamento "não pode ser fornecido nem tampouco realizado para pessoas ou grupos, mas se realiza em processos em que esses se empoderam a si mesmos". Por meio desse processo é possível que as pessoas renunciem a situações de tutela, impotência e dependência, assumindo assim a direção de sua própria vida ${ }^{(10)}$.

As enfermeiras devem promover o empoderamento da mulher a fim de estimular a atuação consciente e resgatar o cuidado centrado nas necessidades da mesma, além de exercer uma prática ética fundamentada em evidências durante as consultas de enfermagem. Tendo desse modo uma postura de influenciadora do auto-cuidado, capaz de estimular que elas reflitam sobre suas situações, afim de nutrilas com o espírito empoderador, e torná-las cada vez mais conscientes de si e de seu corpo. Tornando-as assim protagonistas de suas histórias de vida, superando a relação autoritária entre profissional e usuária de modo que essas mulheres possam tomar decisões com liberdade sobre seu corpo de forma autônoma sem que haja dependência e submissão. E ainda fortalecendo os laços entre enfermeira-paciente no que tange a liberdade de discutir o que é melhor para expressão de sua sexualidade, prevenção de doenças e promoção de sua saúde em geral.

No que tange a realização das enfermeiras em atender as mulheres, sabe-se que quando uma enfermeira cuida de uma mulher, essa profissional também está cuidando indiretamente de todos que estão a volta daquela usuária. Quando esta mulher é bem acolhida por todos os profissionais da Unidade básica de saúde, a comunicação torna-se mais fácil. O que gera confiança por parte da usuária, e se consolida o processo de troca entre cuidador e ser cuidado.

No ponto de vista da enfermagem, cuidar do outro significa atender às suas necessidades com presteza, empatia, sensibilidade e solidariedade, mediante a conduta ética, responsável, crítica e embasada cientificamente de modo que o cuidado realizado possa gerar o conforto e o bem-estar. Sendo imprescindível que durante a consulta de enfermagem a enfermeira tenha uma prática baseada em evidencias e que ao mesmo tempo contemple o cuidado humano, sensível, com carinho e respeito pela outra mulher que está nesse momento frente a ela como cliente. 
As representações sociais de corpo e sexualidade interferem nas práticas de cuidado ofertadas pelos/as profissionais de saúde ${ }^{(11)}$. Quando a enfermeira consegue atingir seu alvo em atender essas mulheres ela sente-se plena consigo mesma, deste modo busca conhecimento para aprofundar-se na profissão e para atender cada vez melhor seu público alvo. Podendo sentir-se realizada profissionalmente, com isso consegue atender melhor todos os que estão a sua volta, trazendo uma cobertura da população feminina mais eficaz.

As questões de gênero também permeiam essa relação de promoção da saúde feminina, pois ainda existem mulheres em situação de vulnerabilidade e submissão ao cônjuge ou outros membros da família do sexo masculino, e esse aspecto pode interferir no tratamento e seguimento dos casos relacionados ao cuidado feminino. As participantes da pesquisa citaram questões relacionadas ao gênero como dificuldades para sua prática junto às mulheres.

Apesar do avanço dos movimentos feministas nas sociedades ocidentais no que tange os direitos sexuais e reprodutivos, o sexo feminino ainda se encontram em ampla desigualdade. Mudanças culturais e estruturais são necessárias para que se alcance a tão sonhada igualdade de direitos entre homens e mulheres ${ }^{(12)}$.

O papel da mulher ainda é de submissão com relação às questões sexuais e a responsabilização das mulheres no que diz respeito às questões reprodutivas impossibilitam o diálogo com seus cônjuges e elevam sua vulnerabilidade. ${ }^{(12)}$.

\subsection{FALAR SOBRE A FALTA DE RECURSOS NO SUS}

É notório que no SUS a falta de recursos humanos e a dificuldade de acesso aos serviços de saúde, configuram um cenário desafiador para os profissionais de saúde, principalmente na Atenção primaria onde interfere diretamente no bom funcionamento de prestação de serviço para a população que mais necessita, visto que são nas ESF que são realizadas ações de promoção prevenção e recuperação de saúde.

\section{CONCLUSÃO}

As enfermeiras se sentem realizadas e citam que representam socialmente a promoção do cuidado às mulheres atendidas por elas. Se reconhecem como profissional que dirige suas ações para o empoderamento das mulheres mesmo em situações adversas como as questões que gênero que dificultam o cuidado integral, ou questões administrativas que surgem no caminho de seu cuidado integral frente ao público feminino. 
Conclui-se que as enfermeiras valorizam seu trabalho no cuidado às mulheres e ainda se sentem responsáveis pela educação em saúde e a transformação no dia a dia das clientes para a consolidação dos princípios do SUS e de condições de saúde individual e coletiva no cotidiano de suas clientes.

\section{REFERENCIAS}

1 Brasil. Ministério da Saúde. Saúde da Família: uma estratégia para reorientação do modelo assistencial. Brasília, 1998 [Internet]. [cited 2018 Apr]. Available from: http://bvsms.saude.gov.br/bvs/publicacoes/cd09_16.pdf

2 Secretaria Nacional De Políticas Para As Mulheres. Monitoramento e acompanhamento da política nacional de atenção integral à saúde da mulher e do plano nacional de políticas para as mulheres. Brasília, 2015 [Internet]. [cited 2018 Apr]. Available from: http://www.spm.gov.br/central-deconteudos/publicacoes/publicacoes/2015/pnaism_pnpm-versaoweb.pdf

3 Brasil. Ministério da Saúde. Protocolos da Atenção Básica: Saúde das Mulheres / Ministério da Saúde, Instituto Sírio-Libanês de Ensino e Pesquisa - Brasília: Ministério da Saúde, 2016. [Internet]. [cited 2018 Aug]. Available from: http://189.28.128.100/dab/docs/portaldab/publicacoes/protocolo_saude_mulher.pdf

4 Moussatché H, Pernambuco DLC. Moscovici S. A representação social da psicanálise. Rio de Janeiro, Zahar, 1978 [Internet]. [cited 2018 Apr]. Available from: http://emaberto.inep.gov.br/index.php/emaberto/article/view/1958/1927

5 Ferreira MA. Teoria das Representações Sociais e Contribuições para as Pesquisas do Cuidado em Saúde e de Enfermagem. Escola Anna Nery, Rio de Janeiro. Junho de 2016 [Internet]. [cited 2018 Apr 28]. Available from: http://www.scielo.br/pdf/ean/v20n2/1414-8145-ean-20-02-0214.pdf

6 Silva SED, Santos AL, Costa JL, Cunha NMF, Araujo JS, Moura AAA . A teoria das representações sociais sob a ótica das pesquisas de enfermagem no Brasil. J Health BiolSci. Jul-Set; 5(3) 2017[Internet]. [cited 2018 Sept]. Available from: http://www.scielo.br/pdf/reben/v64n5/a22v64n5.pdf

7 Câmara RH. Análise de conteúdo: da teoria à prática em pesquisas sociais aplicadas às organizações. Gerais: Revista Interinstitucional de Psicologia, 6 (2), jul - dez, 2013,179-191

8 Nunes CM, Tronchin DMR, Melleiro MM, Kurcgant P. Satisfação e insatisfação no trabalho na percepção de enfermeiros de um hospital universitário. Rev. Eletr. Enf. [Internet] 2010;12(2):252-[cited 2018 Oct]. Available from: https://www.fen.ufg.br/revista/v12/n2/v12n2a04.htm.

9 Reis AD, Santos RD, Paschoal AJ. O cuidado à mulher na contemporaneidade: reflexões teóricas para o exercício da enfermagem transcultural. Rev. Mineira de Enfermagem. 2012[Internet]. [cited 2018 Sept 15]. Available from: http://www.reme.org.br/artigo/detalhes/510.

10 Landerdahl MC, Vieira LB, Cortes LF, Padoin SMM. Processo de empoderamento feminino mediado pela qualificação para o trabalho na construção civil. Esc. Anna Nery. 2013;17(2):306-312

11 Belém JM, Alves MJH, Pereira EV, Maia ER, Quirino GS, Albuquerque GA. Prostituição e saúde: representações sociais de enfermeiros/as da Estratégia Saúde da Família. Rev baiana enferm. 2018:32:e25086 [Internet]. [cited 2018 Nov]. Available from: https://portalseer.ufba.br/index.php/enfermagem/article/view/25086 
12 Santos NJS. Mulher e negra: dupla vulnerabilidade às DST/HIV/AIDS.Rev saúde e sociedade. 25 (3) Jul-Sep 2016 [Internet]. [cited 2018 Nov]. Available from: http://www.scielo.br/scielo.php?pid=S0104$12902016000300602 \&$ script $=$ sci_abstract\&tlng=pt 